\title{
The effect of intrinsic birefringence in deep UV-lithography
}

\author{
Alexander Serebriakov ${ }^{* a}$, E. Maksimov ${ }^{b}$, Florian Bociort ${ }^{\mathrm{a}}$, Joseph Braat ${ }^{\mathrm{a}}$ \\ ${ }^{a}$ Optics Research Group, Delft Univ. of Technology, Lorentzweg 1, 2628 CJ Delft, The Netherlands; \\ ${ }^{b}$ P.N.Lebedev Physical Institute, Leninskiy pr. 53, Moscow 119991, Russia
}

\begin{abstract}
The subject of birefringence induced by spatial dispersion (BISD), also called intrinsic birefringence, recently became an important issue for 157-nm lithography. For the deep UV range, because of intrinsic absorption, only crystalline materials can be used as optical materials for lens manufacturing. The physical properties of crystals are basically affected by spatial dispersion, especially at very short wavelengths. The resulting BISD leads to a serious deterioration of optical image quality.

Recently the mathematical formalism for analyzing those aspects of the BISD effect that are relevant for optical design has been published. In this work we give an equivalent but simplified derivation of these results. This mathematical formalism is then applied to optical system design and the correction methodology is discussed. An example of optical system is given that has been corrected for the BISD effect.
\end{abstract}

Keywords: lithography, birefringence, crystal, optical system design

\section{INTRODUCTION}

The phenomenon of birefringence in certain types of crystals has been known for more than three centuries. It was first discovered by Bartholinus, qualitatively explained by Huygens and marvellously described quantitatively by Fresnel. A firm foundation for the phenomenon of birefringence was obtained by applying Maxwell's laws to crystalline media with specific symmetry properties. In this classical description, cubic crystals do not show birefringence and, indeed, for most purposes they can effectively be considered as being isotropic.

It was Lorentz who first indicated the presence of anisotropy introduced by spatial dispersion in cubic crystals as early as 1878 [1]. This observation was made again in [2] on the basis of a microscopic investigation of quadripolar transitions in crystals, and in [3] on the basis of macroscopic electrodynamics. The detailed analysis of this problem has been carried out in the first edition of Ref. [4]. It was shown, among others, that cubic crystals possess seven optical axes (the three main crystallographic axes and the four body diagonals of the cube). In this respect cubic crystals can be called heptaxial.

A number of experimental and theoretical studies were carried out in the 1970's on the birefringence effect induced by spatial dispersion in semiconductors (see [4], Section 4.6.2). Because of the very small size of the effect and the absence of any practical applications these investigations have not been done in much detail.

Recent publications by Burnett et al demonstrate the great practical importance of the phenomenon [5,6]. The birefringence induced by spatial dispersion has been measured and calculated for $\mathrm{CaF}_{2}$ and $\mathrm{BaF}_{2}$ in the ultraviolet part of the spectrum. It was shown that the magnitude of the BISD in these cubic crystals is sufficiently large to cause serious problems when using $\mathrm{CaF}_{2}$ for precision UV optical systems at wavelengths as short as $157 \mathrm{~nm}$ [6]. The birefringence effect is extensively described and quantified in these references. However some aspects would profit from a further analysis. In Section 2 of this paper we present a different theoretical approach to the BISD subject which allows us to simplify the derivations and to obtain fully equivalent analytic expressions of the final formulae for cubic crystals

*a.serebriakov@tnw.tudelft.nl; phone+31 152784250; fax +31 152788105 
(Section 3). In the final fourth Section we present our calculation method for the BISD and the compensation of its detrimental effect on the image quality in a lithographic objective.

\section{BIREFRINGENCE IN CRYSTALLINE MEDIA}

We will use the macroscopic electrodynamic approach [4] to analyze the birefringence in crystals. The macroscopic Maxwell equations for the electromagnetic field quantities in a medium are written as

$$
\begin{gathered}
\operatorname{rot} \mathbf{B}=\frac{1}{c} \frac{\partial \mathbf{D}}{\partial t}+\frac{4 \pi}{c} \mathbf{j}^{e x t} \\
\operatorname{div} \mathbf{D}=4 \pi \rho^{e x t} \\
\operatorname{rot} \mathbf{E}=-\frac{1}{c} \frac{\partial \mathbf{B}}{\partial t} \\
\operatorname{div} \mathbf{B}=0 .
\end{gathered}
$$

Here $\mathbf{E}$ is the electric field strength, $\mathbf{D}$ and $\mathbf{B}$ are the electric and magnetic induction. The quantities $\mathbf{j}^{\text {ext }}$ and $\rho^{\text {ext }}$ are the external current and charge densities which are sources of the external electromagnetic field. These equations are supplemented by the relation between the electric induction $\mathbf{D}$ and the electric field $\mathbf{E}$. This material equation can be written in the framework of macroscopic electrodynamics in the following general form

$$
D_{i}(\mathbf{r}, t)=\int_{-\infty}^{t} d t^{\prime} \int d \mathbf{r}^{\prime} \varepsilon_{i j}\left(t-t^{\prime}, \mathbf{r}-\mathbf{r}^{\prime}\right) E_{j}\left(\mathbf{r}^{\prime}, t^{\prime}\right)
$$

where $\varepsilon_{i j}(t, \mathbf{r})$ is the dielectric tensor of the medium.

Here we have used the principle of causality according to which the induction at time $t$ is only determined by the present field and the field at previous times $t^{\prime} \leq t$.

We Fourier transform the Eqs. (1) and (2) assuming that

$$
E_{i}(\mathbf{r}, t)=\int E_{i}(\omega, \mathbf{k}) e^{i(\mathbf{k r}-\omega t)} d \omega d \mathbf{k}
$$

For the other quantities we use the same notation as well. We then obtain

$$
\begin{gathered}
\mathbf{D}(\omega, k)=-\frac{c}{\omega}(\mathbf{k} \times \mathbf{B}(\omega, \mathbf{k})), \\
\mathbf{k} \cdot \mathbf{D}(\omega, k)=0, \\
\mathbf{B}(\omega, \mathbf{k})=\frac{c}{\omega}(\mathbf{k} \times \mathbf{E}(\omega, \mathbf{k})), \\
\mathbf{k} \cdot \mathbf{B}(\omega, k)=0,
\end{gathered}
$$


and

$$
D_{i}(\omega, \mathbf{k})=\varepsilon_{i j}(\omega, \mathbf{k}) E_{j}(\omega, \mathbf{k})
$$

where summation over the index $j$, appearing twice, is assumed.

We can also introduce the inverse dielectric function matrix $\varepsilon_{i j}^{-1}(\omega, \mathbf{k})$ and write

$$
E_{i}(\omega, \mathbf{k})=\varepsilon_{i j}^{-1}(\omega, \mathbf{k}) D_{j}(\omega, \mathbf{k})
$$

Writing Eqs. (4)-(7) we have neglected the external currents and charges because they are not important for the main goal of our investigation, namely the dependence of the dielectric function matrix on the wave vector $\mathbf{k}$ taking into account the spatial dispersion effect. The spatial dispersion is determined by the parameter $a \mathbf{k}$ or by the somewhat more descriptive parameter $a / \lambda$, where $a$ is a characteristic dimension (the radius of "the region of influence", radius of molecular action, etc). and $\lambda$ is the wavelength of the electromagnetic field. In a condensed non-metallic medium the radius $a$ is about the order of the lattice constant. Therefore, the parameter $a / \lambda$ is very small, even in the optical or ultraviolet range of the electromagnetic spectrum.

Eliminating the magnetic induction B from the Eqs. (4) - (7) we obtain the expression

$$
\mathbf{D}=-\frac{c^{2}}{\omega^{2}}[\mathbf{k} \times(\mathbf{k} \times \mathbf{E})]=\frac{c^{2}}{\omega^{2}}\left[k^{2} \mathbf{E}-\mathbf{k} \cdot(\mathbf{k} \cdot \mathbf{E})\right],
$$

and, substituting the Eq. (8) into (10), we find

$$
\left[\frac{\omega^{2}}{c^{2}} \varepsilon_{i j}(\omega, \mathbf{k})-k^{2}\left(\delta_{i j}-\frac{k_{i} k_{j}}{k^{2}}\right)\right] E_{j}(\omega, \mathbf{k})=0 .
$$

If we use the matrix $\varepsilon_{i j}^{-1}(\omega, \mathbf{k})$ we obtain

$$
\left[\frac{\omega^{2}}{c^{2}} \delta_{i j}-k^{2}\left(\delta_{i l}-\frac{k_{i} k_{l}}{k^{2}}\right) \varepsilon_{l j}^{-1}(\omega, \mathbf{k})\right] D_{j}(\omega, \mathbf{k})=0
$$

These homogeneous systems of algebraic equations have nontrivial solutions $\mathbf{E}(\omega, k) \neq 0$ and $\mathbf{D}(\omega, k) \neq 0$, only if the corresponding determinants vanish

$$
\left|\frac{\omega^{2}}{c^{2}} \varepsilon_{i j}(\omega, \mathbf{k})-k^{2}\left(\delta_{i j}-\frac{k_{i} k_{j}}{k^{2}}\right)\right|=0,
$$

and

$$
\left|\frac{\omega^{2}}{c^{2}} \delta_{i j}-k^{2}\left(\delta_{i l}-\frac{k_{i} k_{l}}{k^{2}}\right) \varepsilon_{l j}^{-1}(\omega, \mathbf{k})\right|=0 .
$$


The dispersion equations (13) and (14) give the relation between $\omega$ and $\mathbf{k}$ for the electromagnetic normal waves (or eigenwaves) in a given medium for $\omega_{l}=\omega_{l}(\mathbf{k})$ when $l=1,2, \ldots$, where the subscript corresponds to the given normal wave. For these waves, assuming that they are normal, we can write the wave vector $\mathbf{k}$ in the form

$$
\mathbf{k}=\frac{\omega}{c} n(\omega, \mathbf{s}) \mathbf{s}
$$

where $\mathbf{s}$ is the unit vector in the direction $\mathbf{k}$ and $n(\omega, \mathbf{s})$ is the corresponding refraction index. The dispersion equation (13) can be conveniently written in the form

$$
\left|n^{2}(\omega, \mathbf{s})\left(\delta_{i j}-s_{i} s_{j}\right)-\varepsilon_{i j}\left(\omega, \frac{\omega}{c} n(\omega, \mathbf{s}) \mathbf{s}\right)\right|=\varepsilon_{i j} s_{i} s_{j} n^{4}-\left[\left(\varepsilon_{i j} s_{i} s_{j}\right) \varepsilon_{l l}-\varepsilon_{i l} \varepsilon_{l j} s_{i} s_{j}\right] n^{2}+\left|\varepsilon_{i j}\right|=0
$$

This is the fundamental equation of crystal optics. In classical crystal optics $\varepsilon_{i j}=\varepsilon_{i j}(\omega)$ and (16) becomes quadratic with respect to $n^{2}$ and this reduced form is frequently called Fresnel's equation.

For our purpose, it is more convenient to investigate the dispersion equation (14) because we can use the property that the electric induction $\mathbf{D}$ is always transverse for normal waves. It means that we can choose the coordinate system whose $z$-axis is directed along $\mathbf{s}$ and then the vector $\mathbf{D}$ will have only two components $D_{x}$ and $D_{y}$. In the adopted coordinate system the wave equation and the dispersion equation have the following form (taking $s_{1}=s_{2}=0, s_{3}=1$ )

$$
\begin{gathered}
m^{2} D_{\alpha}=\varepsilon_{\alpha \beta}^{-1} D_{\beta}, \\
\left|m^{2} \delta_{\alpha \beta}-\varepsilon_{\alpha \beta}^{-1}\right|=m^{4}-\left(\varepsilon_{11}^{-1}+\varepsilon_{22}^{-1}\right) m^{2}+\varepsilon_{11}^{-1} \varepsilon_{22}^{-1}-\left(\varepsilon_{12}^{-1}\right)^{2}=0
\end{gathered}
$$

in which we introduced the notation

$$
\frac{1}{n^{2}}=m^{2}, \alpha, \beta=1,2
$$

The dispersion equation (18) has two roots for the quantity $m^{2}$

$$
m_{1,2}^{2}=\frac{\varepsilon_{11}^{-1}+\varepsilon_{22}^{-1}}{2} \pm \frac{1}{2} \sqrt{\left(\varepsilon_{11}^{-1}-\varepsilon_{22}^{-1}\right)^{2}-4\left(\varepsilon_{12}^{-1}\right)^{2}}
$$

and, consequently, we are led to two mutually orthogonal vectors $\mathbf{D}_{\mathbf{1}}$ and $\mathbf{D}_{\mathbf{2}}$. As it is well-known from [4] and [7], the existence of two values of the refractive index $n$ for a given direction of the wave vector $\mathbf{k}$ is the origin of the appearance of the birefringence effect. The dispersion equation (18) has a multiple root $m_{1}^{2}=m_{2}^{2}$ if

$$
\left(\varepsilon_{11}^{-1}-\varepsilon_{22}^{-1}\right)^{2}-4\left(\varepsilon_{12}^{-1}\right)^{2}=0
$$

and the birefringence is absent in this case. Also it is well-known from [4] and [7] that in the case of classical crystal optics, i.e. for $\varepsilon_{i j}=\varepsilon_{i j}(\omega)$, the multiple root for $m$ exists for every direction of $\mathbf{k}$ only in the case of cubic crystals. For all other crystals with a lower symmetry, birefringence is absent only for the wave orthogonal to the principal optical axis. For hexagonal, tetragonal or trigonal crystals there is one such an axis and these crystals are called uniaxial. For the 
three remaining crystal systems, namely the orthorhombic, monoclinic and triclinic ones, there are two privileged normal wave directions for which there is no birefringence and the crystals are called biaxial. The situation in this respect is more complicated when the spatial dispersion is taken into account, i.e. if the dielectric function matrix depends on both the frequency $\omega$ and the wave vector $\mathbf{k}$.

\section{SPATIAL DISPERSION OF CUBIC CRYSTALS}

First of all we write the inverse dielectric matrix for cubic crystals in the case of small spatial dispersion in the usual form

$$
\varepsilon_{i j}^{-1}(\omega, \mathbf{k})=\varepsilon_{i j}^{-1}(\omega) \delta_{i j}+\beta_{i j l m}(\omega) k_{l} k_{m}
$$

or as

$$
\varepsilon_{i j}^{-1}(\omega, \mathbf{k})=\varepsilon_{i j}^{-1}(\omega) \delta_{i j}+\beta_{i j l m}(\omega) \frac{\omega^{2}}{c^{2}} n^{2} s_{l} s_{m}
$$

The fourth-rank tensor $\beta_{i j l m}$ has only three independent and non-zero components for cubic crystals with symmetry classes $O, T_{d}$ and $O_{h}[4,8]$. These are

$$
\begin{gathered}
\beta_{1}=\beta_{x x x x}=\beta_{y y y y}=\beta_{z z z z}, \\
\beta_{2}=\beta_{x x z z}=\beta_{y y x x}=\beta_{z z y y}=\beta_{z z x x}=\beta_{x x y y}=\beta_{y y z z}, \\
\beta_{3}=\beta_{x y x y}=\beta_{y z y z}=\beta_{z x z x} .
\end{gathered}
$$

Using these expressions the matrix elements of $\varepsilon_{i j}^{-1}$ can be written as

$$
\begin{gathered}
\varepsilon_{x x}^{-1}=\varepsilon^{-1}(\omega)+\left(\frac{\omega}{c} n\right)^{2}\left[\beta_{1} s_{x}^{2}+\beta_{2}\left(s_{y}^{2}+s_{z}^{2}\right)\right], \\
\varepsilon_{x y}^{-1}=2\left(\frac{\omega}{c} n\right)^{2} \beta_{3} s_{x} s_{y}, \\
\varepsilon_{y y}^{-1}=\varepsilon^{-1}(\omega)+\left(\frac{\omega}{c} n\right)^{2}\left[\beta_{2}\left(s_{x}^{2}+s_{z}^{2}\right)+\beta_{1} s_{y}^{2}\right], \\
\varepsilon_{x z}^{-1}=2\left(\frac{\omega}{c} n\right)^{2} \beta_{3} s_{x} s_{z}, \\
\varepsilon_{z z}^{-1}=\varepsilon^{-1}(\omega)+\left(\frac{\omega}{c} n\right)^{2}\left[\beta_{2}\left(s_{x}^{2}+s_{y}^{2}\right)+\beta_{1} s_{z}^{2}\right],
\end{gathered}
$$




$$
\varepsilon_{y z}^{-1}=2\left(\frac{\omega}{c} n\right)^{2} \beta_{3} s_{y} s_{z}
$$

The factor two in the expressions for $\varepsilon_{x y}^{-1}, \varepsilon_{x z}^{-1}$ and $\varepsilon_{y z}^{-1}$ is due to the summation in (22). The equations (22) - (32) allow us to write the expression for the inverse dielectric matrix in the form

$$
\varepsilon_{i j}^{-1}\left(\omega, \frac{\omega}{c} n \mathbf{s}\right)=\left(\varepsilon^{-1}(\omega)+\frac{\omega^{2}}{c^{2}} n^{2} \beta_{2}\right) \delta_{i j}+\frac{\omega^{2}}{c^{2}} n^{2} \tilde{\beta} s_{i}^{2} \delta_{i j}+2 \frac{\omega^{2}}{c^{2}} n^{2} \beta_{3} s_{i} s_{j},
$$

where

$$
\tilde{\beta}=\beta_{1}-\beta_{2}-2 \beta_{3}
$$

The first term in the expression (33) is the isotropic contribution, the second one is anisotropic, but it is expressed in terms of a diagonal matrix; the last term is purely longitudinal. If we rewrite the wave equation (14)

$$
\left[\frac{\delta_{i j}}{n^{2}(\omega, \mathbf{s})}-\left(\delta_{i l}-s_{i} s_{l}\right) \mathcal{E}_{l j}^{-1}\left(\omega, \frac{\omega}{c} n \mathbf{s}\right)\right] D_{j}\left(\omega, \frac{\omega}{c} n \mathbf{s}\right)=0,
$$

it follows after some simple algebra that the longitudinal part of $\varepsilon_{i j}^{-1}$ will disappear from this equation due to the prefactor $\left(\delta_{i l}-s_{i} s_{l}\right)$, because the multiplication of this prefactor with the longitudinal part yields zero.

We will solve Eq. (35) with the aid of perturbation theory and rewrite this equation as

$$
\left(L_{0}+L_{1}\right)_{i j} D_{j}=\rho(\omega) \delta_{i j} D_{j}
$$

and

$$
\rho(\omega)=\rho_{0}(\omega, \mathbf{s})+\rho_{1}(\omega, \mathbf{s})
$$

where

$$
\rho(\omega)=\frac{1}{n^{2}(\omega, \mathbf{s})}
$$

is the eigenvalue of this equation which should be calculated up to the first order perturbations described by the perturbation operator $L_{1}$. It has the form

$$
\left(L_{1}\right)_{i j}=\frac{\omega^{2}}{c^{2}} n^{2}(\omega, \mathbf{s}) \tilde{\beta} s_{i}^{2} \delta_{i j}-\frac{\omega^{2}}{c^{2}} n^{2}(\omega, \mathbf{s}) \tilde{\beta} s_{i} s_{j}
$$

In the framework of perturbation theory, we can change the value $n^{2}(\omega, \mathbf{s})$ in Eq. (39) into $n_{0}^{2}(\omega)$ defined by the zero-order approximation. This zero-order perturbation is defined by the equation

$$
\left(L_{0}\right)_{i j} D_{j}^{0}=\rho_{0}(\omega) D_{i}^{0}, \text { where }
$$




$$
\left(L_{0}\right)_{i j}=\left(\varepsilon^{-1}(\omega)+\frac{\omega^{2}}{c^{2}} n_{0}^{2} \beta_{2}\right)\left(\delta_{i j}-s_{i} s_{j}\right)
$$

and, consequently, Eq. (40) for the zero-order approximation can be written as

$$
\left[\left(\varepsilon^{-1}(\omega)+\frac{\omega^{2}}{c^{2}} n_{0}^{2} \beta_{2}\right)\left(\delta_{i j}-s_{i} s_{j}\right)\right] D_{j}^{0}=\rho_{0}(\omega) D_{i}^{0} .
$$

By using Eq. (5) this equation reduces to the wave equation for isotropic media, which has the multiple root for $\rho_{0}(\omega)$ equal to

$$
\rho_{0}(\omega)=\frac{1}{n_{0}^{2}(\omega)}=\varepsilon^{-1}(\omega)+\frac{\omega^{2}}{c^{2}} n_{0}^{2}(\omega) \beta_{2}
$$

Moreover, in the framework of macroscopic electrodynamics we can neglect the second term in the right part of (43) because there are no possible experiments which can help us to distinguish the terms $\varepsilon^{-1}(\omega)$ and $\frac{\omega^{2}}{c^{2}} n_{0}^{2}(\omega) \beta_{2}$. It means that for the refraction index $n_{0}(\omega)$ in the zero-order approximation we can write

$$
n_{0}^{2}(\omega)=\varepsilon(\omega)
$$

where

$$
\varepsilon(\omega)=\left(\varepsilon^{-1}(\omega)\right)^{-1}
$$

The existence of the multiple root of $\rho_{0}(\omega)$ in the zero-order approximation tells us that the system of equations (36) and (37) has a degenerate kernel [9] and we should use for the calculation of the first order correction to $\rho_{1}(\omega)$ a socalled secular equation. This equation will give also the two correct values of $\tilde{D}_{i}^{0}(\omega, n(\omega, \mathbf{s}))$ resulting from the removing of the degeneracy due to the perturbation operator $L_{l}$. If we write the first order correction $\rho_{1}(\omega)$ as

$$
\rho_{1}(\omega)=\frac{\omega^{2}}{c^{2}} n_{0}^{2}(\omega) \tilde{\beta} \tilde{\rho}_{1}(\omega, \mathbf{s}),
$$

the secular equation will have the form

$$
\left(s_{i}^{2} \delta_{i j}-s_{i} s_{j}\right) e_{j}(\mathbf{s})=\tilde{\rho}(\omega) e_{i}(\mathbf{s})
$$

$$
\sim^{0}
$$

where we introduced the unit vector $\mathbf{e}$ in the direction of $\tilde{\mathbf{D}}(\mathbf{s})$. Moreover, because the vector $\tilde{\mathbf{D}}$ (s) is orthogonal to the vectors s, Eq. (47) can be simplified to

$$
s_{i}^{2} e_{i}(\mathbf{s})=\tilde{\rho}_{1}(\omega, \mathbf{s}) e_{i}(\mathbf{s}) .
$$


$\sim(1,2)$

The two solutions $\rho_{1} \quad$ turn out to be the roots of the quadratic equation

$$
\tilde{\rho_{1}^{2}}(\mathbf{s})-2\left(s_{x}^{2} s_{y}^{2}+s_{x}^{2} s_{z}^{2}+s_{y}^{2} s_{z}^{2}\right) \tilde{\rho_{1}}(\mathbf{s})+3 s_{x}^{2} s_{y}^{2} s_{z}^{2}=0
$$

More details about this step of the derivation will be given in a subsequent paper. Basically, the pair of eigenmodes $\left(e_{1}(\mathbf{s}), e_{2}(\mathbf{s})\right)$ has been chosen in such a way that in this basis the $2 \times 2$ matrix associated to the operator $L_{l}$ becomes diagonal. The two solutions for $\tilde{\rho}_{1}(\omega, \mathbf{s})$ i.e. the diagonal element of the matrix mentioned above are

$$
\tilde{\rho}_{1}^{(1,2)}(\mathbf{s})=\left(s_{x}^{2} s_{y}^{2}+s_{x}^{2} s_{z}^{2}+s_{y}^{2} s_{z}^{2}\right) \pm \sqrt{\left(s_{x}^{2} s_{y}^{2}+s_{x}^{2} s_{z}^{2}+s_{y}^{2} s_{z}^{2}\right)^{2}-3 s_{x}^{2} s_{y}^{2} s_{z}^{2}}
$$

It is obvious that the equation for $\tilde{\rho}_{1}(\omega, \mathbf{s})$ has only one solution for the mentioned above seven directions of the propagation vector $\mathbf{s}$, that are the three main crystallographic axes and the four body diagonals of the cube.

We can also obtain the expression for the eigenmodes of Eq. (48). The components of the eigenmode $e_{2}(\mathbf{s})$, which $\sim(2)$

correspond to the eigenvalue $\rho_{1}$ (s) have the following form

$$
\begin{gathered}
e_{2 z}(\mathbf{s})=\frac{1}{\sqrt{2}} \sqrt{s_{x}^{2}+s_{y}^{2}+\frac{s_{x}^{2} s_{y}^{2}\left(s_{x}^{2}+s_{y}^{2}\right)-\left(s_{x}^{4}+s_{y}^{4}\right) s_{z}^{2}}{r}}, \\
e_{2 x}(\mathbf{s})=e_{2 z}(\mathbf{s}) \frac{s_{y}^{2}\left(s_{z}^{2}-s_{x}^{2}\right)+r}{s_{x} s_{z}\left(s_{x}^{2}-s_{y}^{2}\right)}, \\
e_{2 y}(\mathbf{s})=e_{2 z}(\mathbf{s}) \frac{s_{x}^{2}\left(s_{z}^{2}-s_{y}^{2}\right)+r}{s_{y} s_{z}\left(s_{y}^{2}-s_{x}^{2}\right)},
\end{gathered}
$$

where $r$ is

$$
r=\sqrt{\left(s_{x}^{2} s_{y}^{2}+s_{x}^{2} s_{z}^{2}+s_{y}^{2} s_{z}^{2}\right)^{2}-3 s_{x}^{2} s_{y}^{2} s_{z}^{2}}
$$

For certain directions $\mathbf{s}$ zero denominators appear and then these expressions cannot be used directly. However analytical expressions can be derived for these special cases. For instance for $s_{x}>0$ and $s_{y}>0$, but $s_{x}$ very small we obtain

$$
e_{2 x}(\mathbf{s})=-\sqrt{\frac{1}{s_{z}^{2}}} s_{z}, \quad e_{2 y}(\mathbf{s})=\frac{s_{x} \sqrt{\frac{1}{s_{z}^{2}}} s_{z}}{2 \sqrt{1-s_{z}^{2}}}, \quad e_{2 z}(\mathbf{s})=\frac{1}{2} s_{x} \sqrt{\frac{1}{s_{z}^{2}}}
$$


The components of the eigenmode $e_{1}(\mathbf{s})$ corresponding to the eigenvalue $\rho_{1}(\mathbf{s})$ can be obtained from the equation

$$
e_{1}(\mathbf{s})=e_{2}(\mathbf{s}) \times \mathbf{s}
$$

Our first order correction to the eigenvalue $\tilde{\rho}_{1}(\omega, \mathbf{s})$ coincides with that obtained by Burnett et al in [6]. We have also compared our results produced by Eqs. (51)-(54) and the ones obtained with the Fortran code by Burnett et al from [10] and, in all cases, we have obtained identical results.

If we define the variation of the birefringence with propagation direction according to Eq. (22) in [6] as

$$
\Delta \tilde{\rho}_{1}(\mathbf{s})=\tilde{\rho}_{1}^{(1)}(\mathbf{s})-\tilde{\rho}_{1}^{(2)}(\mathbf{s})=2 \sqrt{\left(s_{x}^{2} s_{y}^{2}+s_{x}^{2} s_{z}^{2}+s_{y}^{2} s_{z}^{2}\right)^{2}-3 s_{x}^{2} s_{y}^{2} s_{z}^{2}}
$$

this value will have the same sign for every direction. The real origin for a possible change of the sign of the birefringence variation shown in Fig. 6 of [6] is related to the behavior of the eigenmodes $e_{1,2}(\mathbf{s})$. This fact can be easily understood using the picture shown in Fig. 1.

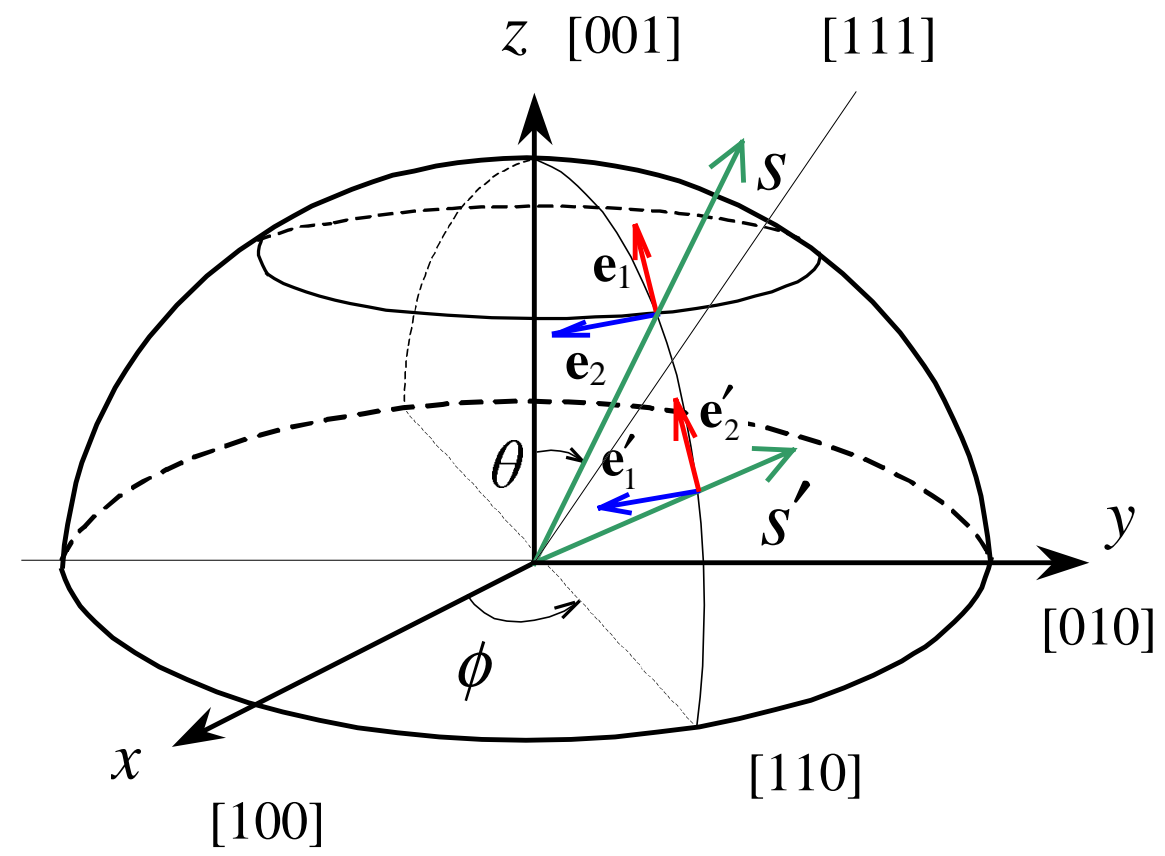

Fig. 1. The behavior of the eigenvectors in the [ $\overline{1} 10]$-diagonal plane of the elementary cubic cell.

Here we show the behavior of the eigenmodes $e_{1,2}(\mathbf{s})$ for two propagation directions in the diagonal plane [ $\left.\overline{1} 10\right]$ of the cubic cell containing the [001], [111] and [110] directions. We can see that the eigenmode $e_{1}(\mathbf{s})$ corresponding to the larger value of $\tilde{\rho}_{1}(\omega, \mathbf{s})$ lies in the plane [ $\left.\overline{1} 10\right]$ for the directions above the axis [111]. The eigenmode $e_{2}(\mathbf{s})$ is normal to this plane. The situation is reverse for the directions below the axis [111]. Here the eigenmode $e_{2}^{\prime}\left(\mathbf{s}^{\prime}\right)$ lies in the plane $[\overline{1} 10]$. It means that if we consider the difference of the refraction indices between one wave polarized in the 
[110] plane and other normal to this plane this difference will have opposite sign for directions above and below axis [111]. This difference has certainly the opposite sign for the vice versa chosen waves as it was shown in Fig. 6 in [6]. Which sign, positive or negative, should be considered as physically correct is not defined. The solution of this dichotomy can be found only considering the process of the refraction of the incoming light with a given polarization on the crystal surface.

\section{BISD COMPENSATION IN DUV LITHOGRAPHY}

In optical system design, BISD leads to the appearance of multiple polarized rays during refraction. There are two basic effects depending on wavelength, thickness and lens shape, which follow from this fact. The first one is the angular difference in ray paths, but due to the minute value of the quantity $\Delta n$ we can neglect it. The second consequence of BISD is the optical pathlength difference between rays of each image bundle (orthogonally polarized). This pathlength difference, resulting in a phase difference, can be visualized in the pupil maps, which are shown in Table 1 for the onesurface case (the dark tone means a small phase difference).

\begin{tabular}{|c|c|c|}
\hline $\begin{array}{c}\text { Chosen } \\
\text { optical axis }\end{array}$ & $\begin{array}{c}\text { The BISD } \\
\text { dependence over } \\
\text { the pupil }\end{array}$ & $\begin{array}{l}\text { The projections of eigenvector states } \\
\text { on the pupil }\end{array}$ \\
\hline$\{100\}$ & & $10=$ \\
\hline$\{110\}$ & & 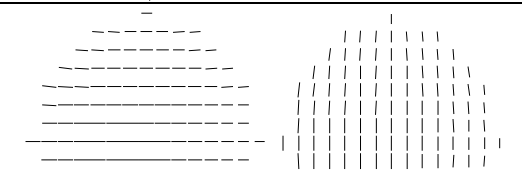 \\
\hline$\{111\}$ & & 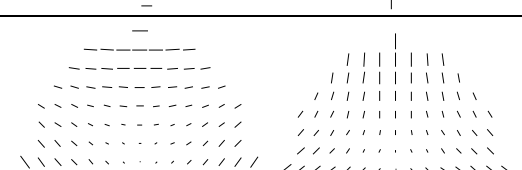 \\
\hline & & 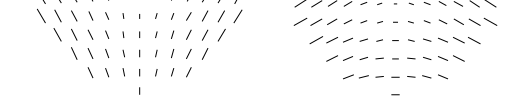 \\
\hline
\end{tabular}

Table 1. Directional dependence of the BISD

The first issue in optical system design with BISD is the calculation of the effect itself. Only one commercial optical design program (Code V, (C) Optical Research Associates) supports the calculation of the effect within a given approximation and allows to analyse the image quality taking into account the BISD. However this application has limitations, e.g. the arbitrary choice of the crystal orientation during optimization is not supported.

The second issue that is also very important is the speed of calculations. For the adequate estimation of the BISD it is necessary to calculate through the system not less than 50 rays taking into account their polarization properties (Table 2). For a lithographic lens with about 50 surfaces it drastically increases the required time for image quality estimation.

Another issue is the search of a relevant characteristic measure of the image quality for BISD in DUV systems. It is very suitable for optical system design to define the influence of BISD on the image quality by one number. In the framework 
of this research it was found that a very good description for the influence of BISD is the standard deviation of the retardation (phase difference) over the pupil. This is confirmed by calculation of BISD in the optical system with and without BISD compensation (Table 3). It is obvious that not only the value of Strehl Ratio but also the standard deviation of the retardation indicates adequately the loss of image quality caused by BISD.

\begin{tabular}{|c|c|c|c|c|c|c|c|c|c|}
\hline \multirow{3}{*}{$\begin{array}{l}\text { Low } \\
\text { BISD }\end{array}$} & Number of rays & 5 & 13 & 24 & 49 & 68 & 89 & 481 & 973 \\
\hline & $\begin{array}{c}\text { Mean } \\
\text { retardation }\end{array}$ & 154.32 & 149.68 & 151.17 & 148.95 & 150.63 & 151.54 & 151.04 & 151.11 \\
\hline & $\begin{array}{c}\text { standard } \\
\text { deviation } \\
\text { of retardation }\end{array}$ & 11.174 & 10.717 & 12.075 & 12.235 & 13.449 & 13.546 & 14.136 & 14.103 \\
\hline \multirow{3}{*}{$\begin{array}{l}\text { High } \\
\text { BISD }\end{array}$} & Number of rays & 5 & 13 & 24 & 49 & 68 & 89 & 481 & 973 \\
\hline & $\begin{array}{c}\text { Mean } \\
\text { retardation }\end{array}$ & 28.176 & 86.253 & 76.489 & 88.437 & 84.640 & 82.043 & 85.119 & 84.729 \\
\hline & $\begin{array}{c}\text { standard } \\
\text { deviation } \\
\text { of retardation }\end{array}$ & 35.734 & 59.455 & 41.456 & 49.023 & 49.291 & 51.595 & 49.944 & 49.791 \\
\hline
\end{tabular}

Table 2. Accuracy of BISD calculation with various numbers of rays

The main obstacle for BISD compensation is the effect of asymmetry with regard to the optical axis. For preserving the optical system symmetry only the three directions $\{100\},\{110\}$ and $\{111\}$ can be selected as an optical axis. The directions $\{100\}$ and $\{110\}$ have an advantage as compare as $\{111\}$ because along them the BISD effect changes less when ray parameters change. However the direction $\{111\}$ is more preferable from the point of view of crystal manufacturing because for this direction the residual stresses are minimal. Generally it is possible to select an arbitrary direction $\{a b c\}$ as an optical axis but in that case the effect calculation and the technological issues can be unpredictably difficult.

\begin{tabular}{|c|c|c|c|c|c|c|c|}
\hline & \multirow{2}{*}{$\begin{array}{c}\text { Strehl Ratio } \\
\text { (in the } \\
\text { Field \# }\end{array}$} & \multicolumn{3}{|c|}{ BISD uncompensated } & \multicolumn{2}{|c|}{ BISD compensation } \\
\cline { 3 - 8 } & BISD) & $\begin{array}{c}\text { Strehl } \\
\text { Ratio }\end{array}$ & $\begin{array}{c}\text { mean } \\
\text { retardation }\end{array}$ & $\begin{array}{c}\text { standard } \\
\text { deviation of } \\
\text { retardation }\end{array}$ & $\begin{array}{c}\text { Strehl } \\
\text { Ratio }\end{array}$ & $\begin{array}{c}\text { mean } \\
\text { retardation }\end{array}$ & $\begin{array}{c}\text { standard } \\
\text { deviation of } \\
\text { retardation }\end{array}$ \\
\hline 1 & 0.7998 & $\mathbf{0 . 4 4 9 8}$ & 77.2561 & $\mathbf{4 8 . 1 6 3 5}$ & $\mathbf{0 . 7 7 6 9}$ & 166.787 & $\mathbf{1 2 . 0 6 3 6}$ \\
\hline 2 & 0.7686 & $\mathbf{0 . 4 2 4 2}$ & 74.4141 & $\mathbf{4 6 . 6 1 9 2}$ & $\mathbf{0 . 7 5 2 6}$ & 162.652 & $\mathbf{1 2 . 5 3 0 7}$ \\
\hline 3 & 0.7734 & $\mathbf{0 . 4 3 2 8}$ & 66.6632 & $\mathbf{4 2 . 2 1 4 9}$ & $\mathbf{0 . 7 5 4 5}$ & 155.734 & $\mathbf{1 3 . 7 3 6 0}$ \\
\hline 4 & 0.7885 & $\mathbf{0 . 4 5 5 0}$ & 58.6569 & $\mathbf{3 7 . 0 9 7 5}$ & $\mathbf{0 . 7 6 8 7}$ & 152.190 & $\mathbf{1 4 . 6 3 1 1}$ \\
\hline 5 & 0.7625 & $\mathbf{0 . 4 4 3 9}$ & 54.8881 & $\mathbf{3 4 . 4 9 6 7}$ & $\mathbf{0 . 7 6 5 1}$ & 153.498 & $\mathbf{1 3 . 0 1 6 5}$ \\
\hline
\end{tabular}

Table 3. Image quality in an optical system with and without BISD compensation

The general strategy of the effect compensation is the following. It can be observed from Table 1 that, being chosen as an optical axis, the direction $\{100\}$ has the $90^{\circ}$ - angular symmetry and $\{111\}$ the $120^{\circ}$ - symmetry. So it is possible to tune the separate components to form the minimal retardation value. However, there is a very important nuance; when we use a $\{111\}$ crystal orientation we should avoid the change of sign of the angle between the incident ray and the optical axis because in this case the orientation $\{111\}-0^{\circ}$ becomes $\{111\}$ - rotated by $60^{\circ}$ and vice versa. The minimum number of components for this method is four $\left(\{100\}-0^{\circ},\{100\}-45^{\circ},\{111\}-0^{\circ},\{111\}-60^{\circ}\right)$. The full effect compensation for the off-axis point is still not clear, as far as the effect behavior is difficult to predict, but in general this behavior is comparable to that of the axial point. 
So the traditional approach implies using the combination of optical components with the optical axes along the crystal orientations $\{001\}$ and $\{111\}$, which can be oriented at different angles with respect to the optical axis. Some combinations of these components result in a more regular phase retardation distribution, which can be adjusted or nulled out with the available optimization tools.

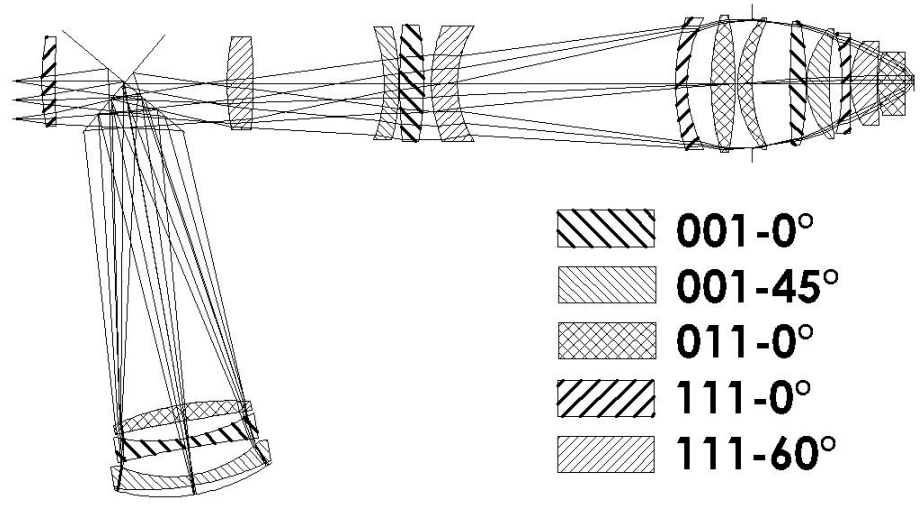

Fig 2. BISD compensation in DUV lithographic system

The novelty of our approach is the addition to this combination of the components with crystal orientation $\langle 011\rangle$. Such an arrangement allows to compensate the effect without or with minor additional optimization and does not break the aberration correction of an already geometrically optimized lens. The optical system designed according to this approach is shown in Fig 2. The image quality characteristics of this system are produced in the Table 3.

\section{CONCLUSIONS}

A simplified mathematical description of BISD has been given in a form that is suitable for optical design. The image quality characteristics and the correction approach have been discussed and an example of an optical system with BISD correction has been presented.

\section{ACKNOWLEDGEMENTS}

We gratefully acknowledge the support of this research by ASM Lithography and we thank John Burnett for helpful discussions.

\section{REFERENCES}

1. H.A. Lorentz, Collected papers, Vol. 2-3. Nijhoff, Den Haag 1936;

2. K.H. Hellwege, Z. Physik 129, p. 626, 1951;

3. V.L. Ginzburg, Sov. Phys. JETR 7, p. 1096, 1958;

4. V. M. Agranovich, V. L. Ginzburg, Kristallooptika s Uchetom Prostranstvennô̂ Dispersii i Teoriya Eksitonov (Crystal Optics with Spatial Dispersion, and Excitons) Nauka, Moscow, 1979 [Translated into English (SpringerVerlag, Berlin, 1984)];

5. John H. Burnett, Zachary H. Levine, and Eric L. Shirley "Intrinsic birefringence in calcium fluoride and barium fluoride", Phys. Review B, 64, p. 241102, 2000;

6. John H. Burnett, Zachary H. Levine, Eric L. Shirley, and John H. Bruning, "Symmetry of spatial-dispersion-induced birefringence and its implications for CaF2 ultraviolet optics", Journal of Microlithography, Microfabrication and Microsystems, 1, p. 213-224, 2002;

7. L.D. Landau, Electrodynamics of continuous media, Pergamon Press, Oxford, 1984;

8. J.F. Nye, Physical properties of crystals, Clarendon Press, Oxford, 1985;

9. R. Courant, Methods of mathematical physics, Vol. 1, Interscience, New York, 1989;

10. http://physics.nist.gov/Divisions/Div842/Gp3/DUVMatChar/DUVbirefring.html 